REV EXP MED 2019;5(2).

Abril - Junio

\title{
Frecuencia y factores asociados a toxocariosis y toxoplasmosis en gestantes admitidas en un hospital del norte del Perú
}

\author{
Nury Miluska Lopez-Gomez 1.a, Becerra-Gutierrez, Lizzie Karen ${ }^{2 b}$, Franklin Rómulo Agui- \\ lar-Gamboa ${ }^{2 a}$; Emma Vanesa Arriaga-Deza ${ }^{3 c}$, Silva-Díaz, Heber ${ }^{3,4 b}$
}

\section{RESUMEN}

Objetivo: Determinar la frecuencia y los factores asociadas a toxocariosis y toxoplasmosis en gestantes admitidas en un hospital del norte del Perú. Materiales y métodos: Se realizó un estudio tipo observacional analítico y prospectivo. La toxocariosis y toxoplasmosis se diagnosticaron mediante la detección de anticuerpos IgM e IgG específicos por la técnica de Elisa. Resultados: Se observó una frecuencia de $10,0 \%(6 / 60)$ y $30,0 \%(18 / 60)$ para toxocariosis y toxoplasmosis, respectivamente. El $28,3 \%$ manifestaron tener antecedentes familiares de aborto, y el $20,0 \%$ y $66,7 \%$ tuvieron contacto con suelo y perros, respectivamente. Conclusiones: La toxoplasmosis y toxocariosis presentan una alta frecuencia en gestantes en la población estudiada, evidenciando un problema de salud pública por atender. Se recomienda su vigilancia y establecer mejoras en el saneamiento y políticas de prevención.

Palabras clave: Toxoplasmosis, toxoxariasis, estudios seroepidemiológicos, embarazo (Fuente: DeCS-BIREME)

\section{Frequency and factors associated with toxocariosis and toxoplas- mosis in pregnant women admitted to a hospital in northern Peru}

\section{ABSTRACT}

Objective: To determine frequency and factors associated to toxocariasis and toxoplasmosis in pregnant women admitted at a northern Peru hospital. Materials and methods: Prospective observational and analytical study. Toxocariasis and toxoplasmosis were diagnosed using ELISA to detect specific IgM and IgG antibodies. Results: $10 \%(6 / 60)$ and $30 \%(20 / 60)$ frequencies for toxocariasis and toxoplasmosis were observed, respectively. $28.3 \%$ manifested to have abortion family records, and the $20 \%$ and $66.7 \%$ were in contact with soil and dogs, respectively. Findings: Toxoplasmosis and toxocariasis present a high frequency in pregnant women, evidencing a public health issue that must be attended. Vigilance, sanitation improvement and prevention policies are recommended.

Key words: Toxoplasmosis, Toxocariosis, Seroepidemiologic Studies, Pregnancy (Source: MESH-NLH

\footnotetext{
1. Facultad de Ciencias Biológicas, Universidad Nacional Pedro Ruiz Gallo, Lambayeque, Perú.

2. Laboratorio de Inmuno-Virología, Hospital Regional Lambayeque, Lambayeque, Perú.

${ }^{3}$ Facultad de Ciencias de la Salud, Universidad Particular de Chiclayo, Lambayeque, Perú.

${ }^{4}$ Dirección de Investigación, Hospital Regional Lambayeque, Lambayeque, Perú.

a. Biólogo.

b. Biólogo, doctor en ciencias.

c Biólogo, maestro en ciencias.
} 


\section{INTRODUCCIÓN}

La toxoplasmosis y toxocariosis son enfermedades zoonóticas causadas por los parásitos Toxoplasma gondi y Toxocara spp., respectivamente ${ }^{(1)}$. La toxoplasmosis en personas inmunocompetentes presenta características clínicas no específicas, sin embargo en inmunocomprometidos produce cuadros clínicos graves ${ }^{(2,3)}$. La infección primaria en el embarazo puede ser trasmitida al feto a través de la placenta, ocasionando afecciones graves e incapacitantes, incluso la muerte (3). De modo similar la toxocariosis en humanos es asintomática; sin embargo, cobra interés cuando las larvas migran hacia diferentes órganos provocando síndromes clínicos como el de la larva migrante visceral, ocular y toxocariosis encubierta (4).

La toxoplasmosis en gestantes es una infección de la cual se conocen los daños fetales, sin embargo, estos aspectos son poco conocidos en la toxocariosis. Existe un solo registro sobre la infección congénita de Toxocara sp. en humanos, el cual ocurrió en un niño prematuro cuya madre era seropositiva para Toxocara sp.(5). La prevalencia de estas infecciones varía ampliamente entre distintos países según las medidas de prevención y condiciones socioeconómicas y ambientales ${ }^{(5,6) ;}$ no obstante, actualmente esta infección parasitaria está emergiendo como importante infección transmitida a través de alimentos ${ }^{(7,8)}$.

La importancia de estas infecciones radica en las consecuencias que éstas producen a corto y largo plazo. Son infecciones parasitarias desatendidas y relacionadas a países en desarrollo, razón por el cual la ausencia de vigilancia epidemiológica permite que no se conozcan su frecuencia real y factores de riesgo asociados a la infección en mujeres embarazadas en el Perú (1).

Por tal motivo, el presente trabajo describió la frecuencia y características asociadas a la toxocariosis y toxoplasmosis en gestantes admitidas en un hospital público del norte del Perú.

\section{MATERIAL Y MÉTODOS}

\section{Tipo y diseño de la investigación}

Estudio observacional analítico y prospectivo. El estudio corresponde a los resultados preliminares de la primera parte de una investigación de mayor complejidad que busca entender la epidemiología de la toxocariosis y toxoplasmosis, tanto en el ámbito hospitalario y comunitario, de mujeres embarazadas.

\section{Población y muestra}

La población estuvo constituida por las gestantes atendidas durante los años 2015 al 2016 en el servicio de obstetricia y ginecología del Hospital Regional Lambayeque (HRL). La muestra fue no probabilística y se consideró a 60 gestantes, que fueron la primera parte de gestantes enroladas del estudio en población hospitalaria. Las participantes fueron seleccionadas por muestreo aleatorio sistemático.

Criterios de inclusión: pacientes en estado de gestación atendidas en el servicio de obstetricia y ginecología del HRL que aceptaron participar voluntariamente en el estudio y accedieron a la firma del consentimiento.

Criterios de exclusión: pacientes gestantes hospitalizadas, toda paciente que presente dificultad mental o condición fisiológica que impida aplicar el cuestionario estructurado o tomar la muestra de sangre requerida.

\section{Técnicas e instrumentos de recolección de datos}

La recolección de los datos se realizó mediante la aplicación de un cuestionario a cada participante, en este de recogieron los posibles factores asociados a las enfermedades, así como otros elementos que nos lleven a un mejor análisis del estudio.

La detección de las enfermedades se realizó a través de la búsqueda de anticuerpos séricos anti-toxoplasma y anti-toxocara en el suero de la paciente, previamente colectado y almacenado a $-70^{\circ} \mathrm{C}$. Las pruebas serológicas realizadas se estandarizaron usando el ensayo inmuno-absorbente ligado a enzima (Elisa, del inglés Enzyme Linked Immunosorbent Assay). Siguiendo las recomendaciones del fabricante (Virion-Serion, Alemania), se evaluaron cuantitativamente anticuerpos IgM e IgG contra $T$. gondii y anticuerpos IgG contra T. canis). La exposición (pasada o reciente) a T. gondii se confirmó por la presencia de anticuerpos lgG o lgM contra el parásito.

\section{Consideraciones éticas}

Para la realización del estudio se contó con el permiso institucional del Hospital Regional Lambayeque y la aprobación del Comité de Ética de Investigación de la misma institución. Se garantizó en todo momento cumplir con los criterios de autonomía y anonimato de cada participante, mediante el consentimiento informado escrito de cada paciente, así como la conservación de los datos colectados bajo salvaguarda de los investigadores.

\section{Análisis estadístico}

Los datos fueron registrados en una base de datos de Microsoft Excel囚 2010. Luego fueron analizados, según la característica de cada variable, con el programa estadístico informático SPSS 20.0. Se realizó un análisis descriptivo de las variables categóricas las cuales fueron presentadas como frecuencias absolutas y relativas. En el análisis bivariado de asociación entre los factores evaluados y la toxoplasmosis o toxocariosis se realizó mediante el test de Chi cuadrado o el test de Fisher exacto, considerando significativo un valor de $p<0,05$. 


\section{RESULTADOS}

Se evaluaron 60 gestantes, las que se caracterizaron por tener una mediana de edad de 23 años (rango intercuartílico de 19 a 30), y una mediana de número de embarazos de tres (rango intercuartílico de 1 a 4). Asimismo, en la muestra se observó que el $18,3 \%$ presentó historia de abortos; $6,7 \%$ tuvo mortinatos y $28,3 \%$ indicó antecedentes familiares de aborto (tabla 1). En cuantos a los hábitos y características ambientales se ob servó que el $20,0 \%$ de la muestra tuvo contacto con el suelo, el 33,3\% consumió agua no potable y el $66,7 \%$ convivió con perros en casa (tabla 2 ).
La frecuencia de seropositividad para anticuerpos IgG contra $T$. canis y $T$. gondii fueron $10,0 \%(6 / 60)$ y $30,0 \%(18 / 60)$, respectivamente. En cuanto al análisis bivariado, ninguno de las factores estudiados en la muestra demostró asociación con la mayor frecuencia de la infección (tablas 1 y 2); sin embargo, se resalta la variable "presencia de perro en casa", como aquella que obtuvo un valor de "p" cercano a la significación $(0,089)$.

Tabla 1. Características del embarazo y antecedentes relacionados en gestantes con toxocariosis y toxoplasmosis.

\begin{tabular}{|c|c|c|c|c|c|}
\hline \multirow[b]{2}{*}{ Variables } & \multirow[b]{2}{*}{$\mathbf{N}(\%)$} & \multicolumn{2}{|c|}{ Toxocariosis } & \multicolumn{2}{|c|}{ Toxoplasmosis } \\
\hline & & Positivo/Total (\%) & p-valor & Positivo/Total (\%) & p-valor \\
\hline \multicolumn{6}{|l|}{ Embarazos } \\
\hline Primeriza & $16(26,7)$ & $0 / 16(0,0)$ & 0,179 & $4 / 16(25,0)$ & 0,755 \\
\hline No Primeriza & $44(73,3)$ & $6 / 44(13,6)$ & & $14 / 44(31,8)$ & \\
\hline \multicolumn{6}{|c|}{ Edad gestacional (trimestral) } \\
\hline Primero & $11(18,3)$ & $1 / 11(9,1)$ & 0,984 & $4 / 11(36,4)$ & 0,878 \\
\hline Segundo & $21(35,0)$ & $2 / 21(9,5)$ & & $6 / 21(28,6)$ & \\
\hline Tercero & $28(46,7)$ & $3 / 28(10,7)$ & & $8 / 28(28,6)$ & \\
\hline \multicolumn{6}{|l|}{ Amenaza Aborto } \\
\hline $\mathrm{Si}$ & $8(13,3)$ & $0 / 8(0,0)$ & 0,585 & $2 / 8(25,0)$ & $>0,900$ \\
\hline No & $52(86,7)$ & $6 / 52(11,5)$ & & $16 / 52(30,8)$ & \\
\hline \multicolumn{6}{|l|}{ Historia de Aborto } \\
\hline $\mathrm{Si}$ & $11(18,3)$ & $0 / 11(0,0)$ & 0,581 & $3 / 11(27,7)$ & $>0,900$ \\
\hline No & $49(81,7)$ & $6 / 49(12,2)$ & & $15 / 49(30,6)$ & \\
\hline \multicolumn{6}{|l|}{ Mortinatos } \\
\hline $\mathrm{Si}$ & $4(6,7)$ & $0 / 4(0,0)$ & $>0,900$ & $2 / 4(50,0)$ & 0,576 \\
\hline No & $56(93,3)$ & $6 / 56(10,7)$ & & $16 / 56(28,6)$ & \\
\hline \multicolumn{6}{|l|}{ Prematuros Vivos } \\
\hline $\mathrm{Si}$ & $9(15,0)$ & $0 / 9(0,0)$ & 0,578 & $3 / 9(33,3)$ & $>0,900$ \\
\hline No & $51(85,0)$ & $6 / 51(11,8)$ & & $15 / 51(29,4)$ & \\
\hline \multicolumn{6}{|c|}{ Antecedentes de aborto familiar } \\
\hline $\mathrm{Si}$ & $17(28,3)$ & $1 / 17(5,9)$ & 0,665 & $5 / 17(29,4)$ & $>0,900$ \\
\hline No & $43(71,7)$ & $5 / 43(11,6)$ & & $13 / 43(30,2)$ & \\
\hline \multicolumn{6}{|l|}{ Anomalía Ocular } \\
\hline $\mathrm{Si}$ & $12(20,0)$ & $1 / 12(8,3)$ & $>0,900$ & $5 / 12(41,7)$ & 0,482 \\
\hline No & $48(80,0)$ & $5 / 48(10,4)$ & & $13 / 48(27,1)$ & \\
\hline
\end{tabular}


Tabla 2. Hábitos y característica ambientales de las gestantes toxocariosis y toxoplasmosis.

\begin{tabular}{|c|c|c|c|c|c|}
\hline \multirow{2}{*}{ Variables } & \multirow{2}{*}{$\mathbf{N}(\%)$} & \multicolumn{2}{|c|}{ Toxocariosis } & \multicolumn{2}{|c|}{ Toxoplasmosis } \\
\hline & & Positivo/Total (\%) & p-valor & Positivo/Total (\%) & p-valor \\
\hline \multicolumn{6}{|c|}{ Consumo carne mal cocida } \\
\hline $\mathrm{Si}$ & $9(15,0)$ & $0 / 9(0,0)$ & 0,578 & $3 / 9(33,3)$ & 1,000 \\
\hline No & $51(85,0)$ & $6 / 51(11,8)$ & & $15 / 51(29,4)$ & \\
\hline \multicolumn{6}{|l|}{ Contacto con el suelo } \\
\hline $\mathrm{Si}$ & $38(63,3)$ & $2 / 38(5,3)$ & 0,179 & $9 / 38(23,8)$ & 0,242 \\
\hline No & $22(36,7)$ & $4 / 22(18,2)$ & & $9 / 22(40,9)$ & \\
\hline \multicolumn{6}{|l|}{ Consumo de agua } \\
\hline Potable & $40(66,7)$ & $3 / 40(7,5)$ & 0,390 & $14 / 40(35,0)$ & 0,370 \\
\hline No Potable & $20(33,3)$ & $3 / 20(15,0)$ & & $4 / 20(20)$ & \\
\hline \multicolumn{6}{|l|}{ Gato en casa } \\
\hline \multicolumn{5}{|r|}{ (10) } & 0,782 \\
\hline No & $29(48,3)$ & $3 / 29(10,3)$ & & $8 / 29(27,6)$ & \\
\hline \multicolumn{6}{|l|}{ Perro en casa } \\
\hline $\mathrm{Si}$ & $40(66,7)$ & $2 / 40(5,0)$ & 0,089 & $13 / 40(32,5)$ & 0,766 \\
\hline No & $20(33,3)$ & $4 / 20(20,0)$ & & $5 / 20(25,0)$ & \\
\hline \multicolumn{6}{|c|}{ Ingestión de vegetales crudos } \\
\hline $\mathrm{Si}$ & $57(95,0)$ & $6 / 57(10,5)$ & $>0,900$ & $18 / 57(31,6)$ & 0,547 \\
\hline No & $3(5,0)$ & $0 / 3(0,0)$ & & $0 / 3(0,0)$ & \\
\hline \multicolumn{6}{|l|}{ Nivel educativo } \\
\hline Primaria & $19(31,7)$ & $2 / 19(10,5)$ & 0,276 & $6 / 19(31,6)$ & 0,942 \\
\hline Secundaria & $33(55,0)$ & $2 / 33(6,1)$ & & $10 / 33(30,3)$ & \\
\hline Superior & $8(13,3)$ & $2 / 8(25,0)$ & & $2 / 8(25,0)$ & \\
\hline
\end{tabular}

\section{DISCUSIÓN}

El presente estudio permitió observar la alta exposición de las gestantes, y mujeres en edad fértil atendidas en el HRL, a los parásitos $T$. canis y $T$. gondii.

Se obtuvo una prevalencia de $10 \%$ de toxocariosis. En el Perú no se encontraron registros similares de esta infección en la misma población estudiada; sin embargo distintos estudios a nivel mundial obtuvieron prevalencias similares con $6,4 \%{ }^{(1)}$. y $9,19 \%{ }^{(10)}$ en Brasil y China respectivamente. Por el contrario en Irán se registró el 21,16\% (4) de prevalencia en gestantes dado que encontraron factores de riesgo asociados a la convivencia con animales, además que la mayor parte de gestantes con la infección provenían de áreas rurales ${ }^{(4)}$. De hecho el presente estudio no presento ningún factor asociado y las gestantes no provenían necesariamente de zona rural.
Por otro lado la frecuencia de toxoplasmosis (infección reciente o antigua) en la población estudiada fue alta $(30,0 \%)$, sin embargo, inferior al reportado por otro estudio en Perú donde obtuvieron prevalencias mayores al

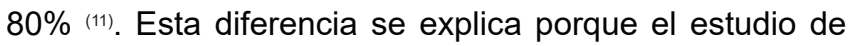
referencia fue realizado en población rural de lquitos, con déficit sanitario y otras características ambientales y sociodemográficas. A nivel mundial se registran diferentes índices de prevalencia de toxoplasmosis en embarazadas, así por ejemplo en Asia registran prevalencia desde $4,5 \%{ }^{(12)}$ a $48 \%{ }^{(13)}$, y en África desde $23.9 \%^{(14)}$ a $24.5 \%{ }^{(15)}$.

Estudios previos han informado sobre los factores asociados a la toxocariosis y toxoplasmosis. La toxocariosis humana fue asociada al contacto con perros ${ }^{(16,9)}$, debido al contacto con huevos de este parásito presentes en las 
heces ${ }^{(10)}$ y piel ${ }^{(17)}$ de sus mascotas. Pese a que en este estudio no se observó asociación entre la tenencia de mascotas con la toxocariosis, probablemente debido al tamaño de muestra. Sin embargo, un estudio observo que la presencia de mascotas es un factor asociado importante ${ }^{(16)}$.

Por otro lado, en cuanto a la toxoplasmosis se han reportado varios factores de riesgo en gestantes: el nivel educativo ${ }^{(18,19)}$, el contacto con gatos ${ }^{(17,18)}$, consumo de agua no potable ${ }^{(14)}$, el consumo de carne cruda o mal cocida y verduras $(19,16,18)$. No obstante, en nuestro estudio ninguno de los factores estudiados estuvo asociado con mayor frecuencia de la toxoplasmosis, debido probablemente al pequeño tamaño de muestra que limitó la fuerza de asociación; no obstante permitió observar la frecuencia de posibles factores como la presencia de perros en casa como aquella que obtuvo un valor de $p$ cercano a la significación. Al respecto, un estudio previo a observado al contacto con perros como factor de riesgo más importante en relación a los gatos, debido a que estos últimos pasan menos tiempo dentro de casa respecto a los canes que poseen una convivencias más directa con el humano (21).

A pesar de que en nuestro estudio no se observó asociación entre el nivel educativo y la presencia de toxoplasmosis un estudio índico que mujeres con alto nivel educativo estuvieron más propensas a adquirir la infección, ya que al generar más ingresos económicos adquirían distintos tipos carnes que eran consumidas sin una adecuada cocción las cuales podrían estar infectadas con bradizoítos ${ }^{(18)}$. Sin embargo, otro estudio revelo que las mujeres con bajo nivel académico se encuentran más expuestas debido a que al generar menos ingresos se dedicaban a otras actividades como la siembra, esto implica el contacto directo con el suelo y por ello mayor exposición a la posible contaminación con ooquistes ${ }^{(10,16,22)}$.

Se resalta el hallazgo de que $28,3 \%$ de gestantes indicó antecedentes familiares de aborto donde la infección de $T$. canis y T. gondii son también causales, razón por el que se deberán considerar en los diagnósticos diferenciales y futuros estudios de investigación. El presente trabajo tuvo limitaciones, principalmente en lo referido al diseño transversal analítico en población hospitalaria y reducido tamaño de muestra no probabilística, los que no permitieron establecer prevalencias generalizables y asociaciones positivas con las variables evaluadas.

Se concluye que la toxocariosis y la toxoplasmosis son frecuentes en gestantes admitidas en el Hospital Regional Lambayeque, lo que indica la importancia de investigar y vigilar estas infecciones en las gestantes y mujeres en edad fértil. Es recomendable tomar medidas de vigilancia de estas enfermedades y establecer mejoras en el saneamiento y políticas de prevención en la población.

Financiamiento. El Hospital Regional Lambayeque a través del uso de la infraestructura y equipamiento de los laboratorios de investigación. Reactivos, insumos de laboratorio y viáticos autofinanciados.

Conflicto de interés. Los autores no poseen conflicto de interés.

\section{REFERENCIAS BIBLIOGRÁFICAS}

1. Adell M, Dea A, Sánchez P, Carmena D. Estudio de las principales zoonosis parasitarias intestinales trasmitidas por perros en la provincia de Catellón y su repercusión en la salud pública. Tesis doctoral. Valencia: Universidad CEU Cardenal Herrera; 2017.

2. Apt W. Infecciones por parásitos más frecuentes y su manejo. Rev Médica Clínica Las Condes. 2014;25(3):485-528.

3. Prusa A-R, Kasper DC, Sawers L, Walter E, Hayde M, Stillwaggon E. Congenital toxoplasmosis in Austria: Prenatal screening for prevention is cost-saving. PLoS Negl Trop Dis. 2017;11(7):1-24.

4. Raissi V, Sohrabi Z, Getso M, Raiesi O, Hafshejani S, Shabandoust H, et al. Risk factors and prevalence of toxocariasis in pregnant women and diabetic patients compared to healthy adults in llam province, western Iran. Excli J. 2018;17:983-938.

5. Maffrand R, Avila-Vázquez M, Princich D, Alasia P. Toxocariosis ocular congénita en un neonato prematuro. Ann Pediatr. 2006;64(6):599600.

6. Peyron F, L'ollivier C, Mandelbrot L, Wallon M, Piarroux R, Kieffer F, et al. Maternal and Congenital Toxoplasmosis: Diagnosis and Treatment Recommendations of a French Multidisciplinary Working Group. Pathogens. 2019;8(1): 24.

7. Hussain MA, Stitt V, Szabo EA, Nelan B. Toxoplasma gondii in the Food Supply. Pathogens. 2017;6(2):21.

8. Choi D, Lim J, Choi D-C, Lee K, Paik S, Kim S-H, et al. Transmission of Toxocara canis via Ingestion of Raw Cow Liver: A Cross-Sectional Study in Healthy Adults. Korean J Parasitol. 2012;50(1):23-27.

9. Santos P, Lehmann L, Lorenzi C, Hirsch C, Telmo P, Mattos G, et al. The Seropositivity of Toxocara spp. Antibodies in Pregnant Women Attented at the University Hospital in Southern Brazil and the Factors Associated with Infection. PLoS One. 2015;10(7):e0131058.

10. Cong W, Zhang X-X, Zhou N, Yu C-Z, Chen J, Wang X-Y, et al. Toxocara Seroprevalence among Clinically Healthy Individuals, Pregnant Women and Psychiatric Patients and Associated Risk Factors in Shandong Province, Eastern China. PLoS Negl Trop Dis . 2014; 8 (8): e3082.

11. Reategui C, Vela L. Factores socioeconómicos-epidemiológicos y su relación con la seroprevalencia de Toxoplasmosis en gestantes atendidasen los hospitales Felipe Arriola y Cesar Garayar, Iquitos, Perú; 2009. Neotrop Helminthol. 2011;5(1):21-40.

12. Smit G, Vu B, Do D, Do Q, Pham H, Speybroeck N, et al. Sero-epidemiological status and risk factors of toxoplasmosis in pregnant women in Northern Vietnam. BMC Infect Dis. 2019;19(1):329.

13. Santos A, Terças A, Ribeiro L, Vasconcellos M, Zenazokenae L, Atanaka M, et al. Serological study on toxoplasmosis in the Haliti-Paresi community of the Utiariti indigenous territory, Campo Novo do Parecis, Mato Grosso, Brazil. Parasite Epidemiol Control. 2019;5:e00097.

14. Jula J, Girones G, Edao B, Deme C, Cebrian J, Butrón L, et al. Seroprevalence of Toxoplasma gondii infection in pregnant women attending antenatal care in southern Ethiopia. Rev Esp Quimioter. 2018;31(4):363-366.

15. Paul E, Kiwelu I, Mmbaga B, Nazareth R, Sabuni E, Maro A, et al Toxoplasma gondii seroprevalence among pregnant women attending antenatal clinic in Northern Tanzania. Trop Med Health. 2018;46(1):39.

16. Pereira LC, Elefant GR, Nóbrega YM, Vital T, Nitz N, Gandolfi L, et al Toxocara spp seroprevalence in pregnant women in Brasília, Brazil. Rev Soc Bras Med Trop. 2016;49(5):641-643. 
17. El-Tras W, Holt $\mathrm{H}$, Tayel A. Risk of Toxocara canis eggs in stray and domestic dog hair in Egypt. Vet Parasitol. 2011;178(3-4):319-323.

18. Bamba S, Cissé M, Sangaré I, Zida A, Ouattara S, Guiguemdé R. Seroprevalence and risk factors of Toxoplasma gondii infection in pregnant women from Bobo Dioulasso, Burkina Faso. BMC Infect Dis.2017;17(1):482.

19. Naheen CR, Tarafder S, Khan S. Toxoplasma gondii Seropositivity and its Possible Associated Risk Factors among Pregnant Women Attending Antenatal Clinic in a Tertiary Care Hospital of Dhaka, Bangladesh. Mymensingh Med J. 2018;27(2):336-343.

20. Jiang R-L, Ma L-H, Ma Z-R, Hou G, Zhao Q, Wu X. Seroprevalence and associated risk factors of Toxoplasma gondii among Manchu pregnant women in northeastern China. Microb Pathog. 2018;123:398-401.

21. Iddawela D, Vithana S, Ratnayake C. Seroprevalence of toxoplasmosis and risk factors of Toxoplasma gondii infection among pregnant women in Sri Lanka: a cross sectional study. BMC Public Health. 2017;17(1):930.

22. Iddawela D, Vithana S, Ratnayake C. Seroprevalence of toxoplasmosis and risk factors of Toxoplasma gondii infection among pregnant women in Sri Lanka: a cross sectional study. BMC Public Health 2017;17(1):930.

Revisión de pares: Recibido: 21/ 05 / 2019 Aceptado: 26/ 06/ 2019 